\title{
Improved ESPRIT Method for Joint Direction-of-Arrival and Frequency Estimation Using Multiple-Delay Output
}

\author{
Wang Xudong, Xiaofei Zhang, Jianfeng Li, and Jinchao Bai \\ College of Electronic and Information Engineering, Nanjing University of Aeronautics and Astronautics, Nanjing 210016, China \\ Correspondence should be addressed to Wang Xudong, xudong@nuaa.edu.cn \\ Received 8 June 2012; Revised 5 July 2012; Accepted 18 July 2012 \\ Academic Editor: Stefano Selleri \\ Copyright () 2012 Wang Xudong et al. This is an open access article distributed under the Creative Commons Attribution License, \\ which permits unrestricted use, distribution, and reproduction in any medium, provided the original work is properly cited.
}

\begin{abstract}
An automatic pairing joint direction-of-arrival (DOA) and frequency estimation is presented to overcome the unsatisfactory performances of estimation of signal parameter via rotational invariance techniques- (ESPRIT-) like algorithm of Wang (2010), which requires an additional pairing. By using multiple-delay output of a uniform linear antenna arrays (ULA), the proposed algorithm can estimate joint angles and frequencies with an improved ESPRIT. Compared with Wang's ESPRIT algorithm, the angle estimation performance of the proposed algorithm is greatly improved. The frequency estimation performance of the proposed algorithm is same with that of Wang's ESPRIT algorithm. Furthermore, the proposed algorithm can obtain automatic pairing DOA and frequency parameters, and it has a comparative computational complexity in contrast to Wang's ESPRIT algorithm. By the way, this proposed algorithm can also work well for nonuniform linear arrays. The useful behavior of this proposed algorithm is verified by simulations.
\end{abstract}

\section{Introduction}

Uniform linear antenna (ULA) arrays have been used in radar, sonar, electron reconnaissance, seismic data processing, and so on [1-4]. The direction-of-arrival (DOA) and frequency estimation of signals impinging on the ULAs are two fundamental problems in array processing [5-10]. The problem of joint DOA and frequency estimation arises in the applications of fields mentioned above. For example, these parameters can be applied to locate the targets for radars and to locate pilot tones in electron reconnaissance systems [11]. Furthermore, a precise estimation of these parameters is helpful to attain a better pulse descriptor word (PDW) and thus enhances the system performance. Optimal techniques based on maximum likelihood [12] are often applicable but might be computationally prohibitive. Some ESPRIT-based joint angle and frequency estimation methods have been proposed in [13-17]. Mathews [13] discuss this problem in the context of radar applications. Pro-ESPRIT is proposed to estimate angle and frequency. Haardt and Nossek [14] discuss the problem in the context of mobile communications for space division multiple access applications. Their method is based on unitary-ESPRIT, which involves a certain transformation of the data to real valued matrices. Multiresolution ESPRIT is used for joint angle frequency estimation in [15]. ESPRIT method is used for frequency and angle estimation under uniform circular array in $[16,17]$. These ESPRIT-based joint angle and frequency estimation methods can give satisfactory estimation performances, but require additional pairing.

The authors in [18] proposed a joint angle and frequency estimation using multiple-delay output based on ESPRIT, which outperform the conventional ESPRIT algorithm, but it requires an additional pairing. The additional pairing fails to work for low signal-to-noise ratio (SNR), which can be shown from the simulation results in Figure 1. Furthermore, the angle estimation of Wang's ESPRIT algorithm can be improved further.

An improved ESPRIT for joint direction-of-arrival (DOA) and frequency estimation is presented in this paper. Compared with Wang's ESPRIT algorithm, the algorithm has the improved performance of DOA estimation. Moreover, the proposed algorithm can obtain automatically paired DOA and frequency estimation, while Wang's ESPRIT 


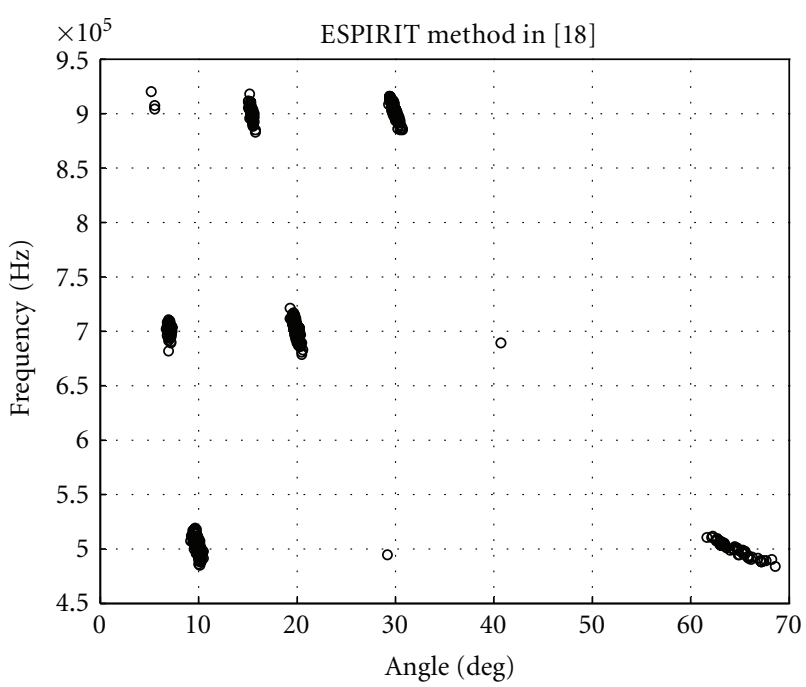

FIGURE 1: Angle-frequency scatter of the Wang's ESPRIT algorithm at $\mathrm{SNR}=0 \mathrm{~dB}$.

algorithm requires the additional pairing. The proposed algorithm has a comparative computational complexity in contrast to Wang's ESPRIT algorithm. Moreover, this proposed algorithm can also work well for nonuniform linear arrays.

Notation 1. $(\cdot)^{T},(\cdot)^{H},(\cdot)^{-1},(\cdot)^{+}$, and $\odot$ denote transpose, conjugate transpose, pseudoinverse operations, and Hadamard product, respectively. Angle $(\cdot)$ refers to the phase of a complex number. $\operatorname{diag}(\mathbf{v})$ stands for diagonal matrix, the diagonal of which is a vector $\mathbf{v}$.

\section{The Data Model}

We consider a uniform linear array with spacing $d$. There is an array of $M$ sensors on which $K$ incident waves impinge $(M>K)$, if the signals are all within the assumed narrow (with respect to the center frequency) band of the receiver, the signal received at the $m$ th antenna is

$$
\begin{array}{r}
y_{m}(t)=\sum_{k=1}^{K} e^{j 2 \pi m d f_{k} \sin \left(\theta_{k}\right) / c} s_{k}(t)+n_{m}(t), \\
m=0,1,2, \ldots, M-1,
\end{array}
$$

where $s_{k}(t)$ is the narrow-band signal of the $k$ th source. $c$ is velocity of light. $\theta_{k}, f_{k}$ are the DOA and the frequency of the $k$ th signal, respectively. $n_{m}(t)$ represents an additive noise term which is assumed to be zero mean and stationary. is

The outputs of the uniform linear antenna arrays (ULA)

$$
\begin{array}{r}
\mathbf{Y}_{0}=\left[y_{0}(n), y_{1}(n), y_{2}(n), \ldots, y_{M-1}(n)\right]^{T}, \\
n=1,2,3, \ldots, N .
\end{array}
$$

Based on this available samples, the problem is to estimate the angles and the frequencies of all sources from the $N$ times snapshots. Suppose the number of signals $K$ is assumed known. Denote the state vector, we get

$$
\mathrm{Y}_{0}=\mathrm{AS}+\mathrm{N}_{0}
$$

where

$$
\begin{gathered}
\mathbf{S}=\left[s_{1}, s_{2}, \ldots, s_{K}\right]^{T} \in \mathbf{C}^{K \times N}, \\
\mathbf{N}=\left[\mathbf{n}_{0}, \mathbf{n}_{1}, \ldots \mathbf{n}_{M-1}\right]^{T} \in \mathbf{C}^{M \times N}, \\
\left.\begin{array}{cccc}
1 & 1 & \cdots & 1 \\
e^{-j \alpha_{1}} & e^{-j \alpha_{2}} & \cdots & e^{-j \alpha_{K}} \\
\vdots & \vdots & \ddots & \vdots \\
e^{-j(M-1) \alpha_{1}} & e^{-j(M-1) \alpha_{2}} & \cdots & e^{-j(M-1) \alpha_{K}}
\end{array}\right],
\end{gathered}
$$

where $\alpha_{k}=2 \pi d f_{k} \sin \left(\theta_{k}\right) / c, k=1,2, \ldots, K$.

In order to joint estimate DOA and frequency, we add $P-1$ delayed outputs for the received signal of array antenna. We suppose that $0<(P-1) \tau<1 / \max \left(f_{i}\right)$.

The delayed signal for (1) with delay $\tau$ is

$$
\begin{aligned}
y_{m}(t-\tau) & =\sum_{k=1}^{K} e^{j 2 \pi m d f_{k} \sin \left(\theta_{k}\right) / c} s_{k}(t-\tau)+n_{m}^{\prime}(t) \\
& =\sum_{k=1}^{K} e^{j 2 \pi m d f_{k} \sin \left(\theta_{k}\right) / c} s_{k}(t) e^{-j 2 \pi f_{k} \tau}+n_{m}^{\prime}(t) .
\end{aligned}
$$

The delayed signal for (5) with $\tau$ can be denoted as

$$
\mathbf{Y}_{1}=\mathbf{A} \Phi \mathbf{S}+\mathbf{N}_{1}
$$

where

$$
\boldsymbol{\Phi}=\operatorname{diag}\left\{e^{-j \beta_{1}}, e^{-j \beta_{2}}, \ldots, e^{-j \beta_{K}}\right\},
$$

where $\beta_{k}=2 \pi f_{k} \tau, k=1,2, \ldots, K$.

The delayed signal for (5) with $p \tau$ can be denoted as

$$
\mathbf{Y}_{p}=\mathbf{A} \Phi^{p} \mathbf{S}+\mathbf{N}_{p}, \quad p=0,1, \ldots, P-1 .
$$

According to (3), (6), and (8), we define

$$
\mathbf{Y}=\left[\begin{array}{c}
\mathbf{Y}_{\mathbf{0}} \\
\mathbf{Y}_{1} \\
\vdots \\
\mathbf{Y}_{P-1}
\end{array}\right]=\left[\begin{array}{c}
\mathbf{A} \\
\mathbf{A} \boldsymbol{\Phi} \\
\vdots \\
\mathbf{A} \Phi^{P-1}
\end{array}\right] \mathbf{S}+\left[\begin{array}{c}
\mathbf{N}_{\mathbf{0}} \\
\mathbf{N}_{1} \\
\vdots \\
\mathbf{N}_{P-1}
\end{array}\right]
$$

\section{Joint Angle and Frequency Estimation}

We can use received signal to attain the direction matrix $\mathbf{A}$ and the delay matrix $\boldsymbol{\Phi}$, and then estimate angle and frequency. The covariance matrix of the received signal can be reconstructed via $\mathbf{R}_{Y}=\mathbf{Y Y}^{H}$. Using eigenvalue decomposition of $\mathbf{R}_{Y}$, we can get the signal subspace $\mathbf{E}_{s}$. In the free-noise case, $\mathbf{E}_{s}$ can be denoted as

$$
\mathbf{E}_{s}=\left[\begin{array}{c}
\mathbf{A} \\
\mathbf{A} \Phi \\
\vdots \\
\mathbf{A} \Phi^{P-1}
\end{array}\right] \mathbf{T}
$$

where $\mathbf{T}$ is a $K \times K$ full-rank matrix. 
3.1. Frequency Estimation. According to (10), we define $\mathbf{E}_{1}$ and $\mathbf{E}_{2}$

$$
\begin{gathered}
\mathbf{E}_{1}=\left[\begin{array}{c}
\mathbf{A} \\
\mathbf{A} \Phi \\
\vdots \\
\mathrm{A} \Phi^{P-2}
\end{array}\right] \mathbf{T}, \\
\mathbf{E}_{2}=\left[\begin{array}{c}
\mathrm{A} \Phi \\
\mathrm{A} \Phi^{2} \\
\vdots \\
\mathrm{A} \Phi^{P-1}
\end{array}\right] \mathbf{T} .
\end{gathered}
$$

According to (11),

$$
\mathbf{E}_{2}=\left[\begin{array}{c}
\mathrm{A} \Phi \\
\mathbf{A} \Phi^{2} \\
\vdots \\
\mathrm{A} \Phi^{P-1}
\end{array}\right] \mathbf{T}=\left[\begin{array}{c}
\mathbf{A} \\
\mathbf{A} \Phi \\
\vdots \\
\mathrm{A} \Phi^{P-2}
\end{array}\right] \mathbf{T T}^{-1} \boldsymbol{\Phi} \mathbf{T}=\mathbf{E}_{1} \mathbf{T}^{-1} \boldsymbol{\Phi} \mathbf{T}
$$

Let $\boldsymbol{\Psi}=\mathbf{T}^{-1} \boldsymbol{\Phi} \mathbf{T}$, so $\boldsymbol{\Psi}=\mathbf{E}_{1}{ }^{+} \mathbf{E}_{2}$. We use eigenvalue decomposition of $\boldsymbol{\Psi}$ to get the estimate of $\boldsymbol{\Phi}$. Also we obtain an estimation of the matrix $\mathbf{T}^{-1}$ via the eigenvectors of $\boldsymbol{\Phi}$. In the no-noise case,

$$
\begin{aligned}
& \widehat{\mathbf{T}}^{-1}=\mathbf{T}^{-1} \Pi \\
& \widehat{\boldsymbol{\Phi}}=\Pi^{T} \boldsymbol{\Phi},
\end{aligned}
$$

where $\Pi$ is a permutation matrix, and $\Pi^{-1}=\Pi^{T}$.

Because $\boldsymbol{\Psi}$ has the same eigenvalues as $\boldsymbol{\Phi}$, we can get $\beta_{k}$, $k=1,2, \ldots, K$ from the eigenvalues $\lambda_{k}, k=1,2, \ldots, K$ of matrix $\boldsymbol{\Psi}$. And then estimate frequency $f_{k}, k=1,2, \ldots, K$

$$
\widehat{f}_{k}=\frac{1}{2 \pi \tau} \operatorname{angle}\left(\lambda_{k}\right)
$$

3.2. Angle Estimation. There exists a transformation matrix $\mathbf{H}$ corresponding to the finite number of row interchanged operations for subspace $\mathbf{E}_{s}$ such that

$$
\mathbf{E}_{c}=\mathbf{H E}_{s} \widehat{\mathbf{T}}^{-1}=\mathbf{H E}_{s} \mathbf{T}^{-1} \boldsymbol{\Pi}=\mathbf{F} \Pi,
$$

where

$$
\mathbf{F}=\mathrm{HE}_{s} \mathbf{T}^{-1}=\mathbf{H}\left[\begin{array}{c}
\mathbf{A} \\
\mathrm{A} \Phi \\
\vdots \\
\mathbf{A} \Phi^{P-1}
\end{array}\right]=\left[\begin{array}{c}
\mathbf{G} \\
\mathbf{G} \Phi \\
\vdots \\
\mathbf{G} \Phi^{M-1}
\end{array}\right]
$$

where

$$
\begin{gathered}
\mathbf{G}=\left[\begin{array}{cccc}
1 & 1 & \cdots & 1 \\
e^{-j \beta_{1}} & e^{-j \beta_{2}} & \cdots & e^{-j \beta_{K}} \\
\vdots & \vdots & \ddots & \vdots \\
e^{-j(P-1) \beta_{1}} & e^{-j(P-1) \beta_{2}} & \cdots & e^{-j(P-1) \beta_{K}}
\end{array}\right], \\
\mathbf{\Phi}=\operatorname{diag}\left\{e^{-j \alpha_{1}}, e^{-j \alpha_{2}}, \ldots, e^{-j \alpha_{K}}\right\} \in \mathbf{C}^{K \times K},
\end{gathered}
$$

where

$$
\alpha_{k}=2 \pi d f_{k} \sin \left(\theta_{k}\right) / c, \quad k=1,2, \ldots, K
$$

According to (16) and (17), take the first $(M-1) P$ rows of $\mathbf{E}_{c}$, we define matrix $\mathbf{E}_{c 1}$ as follows:

$$
\mathbf{E}_{c 1}=\left[\begin{array}{c}
\mathbf{G} \\
\mathbf{G} \Phi \\
\vdots \\
\mathbf{G} \Phi^{M-2}
\end{array}\right] \Pi
$$

Taking the last $(M-1) P$ rows of $\mathbf{E}_{c}$, we define matrix $\mathbf{E}_{c 2}$

$$
\mathbf{E}_{c 2}=\left[\begin{array}{c}
\mathbf{G} \Phi \\
\mathbf{G} \Phi^{2} \\
\vdots \\
\mathbf{G} \Phi^{M-1}
\end{array}\right] \Pi
$$

We define

$$
\mathbf{C}=\mathbf{E}_{c 1}^{+} \mathbf{E}_{c 2} \text {. }
$$

According to (20) and (21), in free-noise case, C can be denoted as

$$
\mathbf{C}=\Pi^{T} \Phi \Pi
$$

Then, the DOA estimation is obtained via

$$
\hat{\theta}_{k}=\arcsin \left(\frac{c}{2 \pi f_{k} d} \operatorname{angle}\left(\xi_{k}\right)\right), \quad k=1,2, \ldots, K,
$$

where $\xi_{k}$ is the $k$ th diagonal element of matrix $\mathbf{C}$.

The following summarizes the major steps of this proposed algorithm.

(1) Compute the covariance matrix from the received signal via $\mathbf{R}_{Y}=\mathbf{Y} \mathbf{Y}^{H}$.

(2) Get the signal subspace $\mathbf{E}_{s}$ by using EVD of $\mathbf{R}_{Y}$. Construct the matrices $\mathbf{E}_{1}$ and $\mathbf{E}_{2}$ from $\mathbf{E}_{s}$ via (11) and (12).

(3) Employ EVD on $\mathbf{E}_{1}{ }^{+} \mathbf{E}_{2}$ to get $\widehat{\mathbf{T}}^{-1}$ and $\hat{\boldsymbol{\Phi}}$. Estimate frequency via (15).

(4) Compute $\mathbf{E}_{c}$ by (16) and construct matrices $\mathbf{E}_{c 1}$ and $\mathbf{E}_{c 2}$ via (20) and (21).

(5) Compute the matrix $\mathbf{C}=\mathbf{E}_{c 1}^{+} \mathbf{E}_{c 2}$ and estimate DOA by (24).

Remark 1. The proposed algorithm can obtain automatically parameter estimation. From (14) and (23), DOA and frequency estimations suffer from the same permutation ambiguity, so we can get automatically paired DOA and frequency. 
3.3. Complexity Analysis. Note that $M, K, N$ and $P$ are the number of the antennas, the sources, the snapshots, and delays, respectively. Computational complexity of Wang's ESPRIT algorithm and this proposed algorithm is a function of $M, K, N$, and $P$.

In contrast to Wang's ESPRIT algorithm, the proposed algorithm has a comparative computational load. For the proposed algorithm, the covariance matrix estimation costs $O\left(M^{2} P^{2} N\right)$; eigen decomposition of $\mathbf{R}_{Y}$ needs $O\left(M^{3} P^{3}\right)$; the computation of $\mathbf{E}_{1}{ }^{+} \mathbf{E}_{2}$ requires $O\left(2 K^{2} M(P-1)+2 K^{3}\right)$; eigen decomposition of $\mathbf{E}_{1}{ }^{+} \mathbf{E}_{2}$ needs $O\left(K^{3}\right)$; compute the matrix $\mathbf{C}=\mathbf{E}_{c 1}^{+} \mathbf{E}_{c 2}$ needs $O\left(2 K^{2}(M-1) P+2 K^{3}\right)$. The major computational complexity of this algorithm is

$$
\begin{aligned}
O & \left(M^{2} P^{2} N+M^{3} P^{3}+\left(2 K^{2} M(P-1)+2 K^{3}+K^{3}\right)\right. \\
& \left.+\left(2 K^{2}(M-1) P+2 K^{3}\right)\right)
\end{aligned}
$$

while Wang's ESPRIT algorithm requires

$$
\begin{aligned}
O & \left(M^{2} P^{2} N+M^{3} P^{3}+\left(2 K^{2} M(P-1)+2 K^{3}+K^{3}\right)\right. \\
& \left.+\left(2 K^{2}(M-1)+2 K^{3}+3 K^{3}\right)\right) .
\end{aligned}
$$

According to [19], we derive the CRB for the frequency estimation and angle estimation as

$$
\mathrm{CRB}=\frac{\sigma^{2}}{2 N}\left\{\operatorname{Re}\left[\mathbf{D}^{H} \boldsymbol{\Pi}_{\mathbf{A}}^{\perp} \mathbf{D} \odot \hat{\mathbf{P}}_{s}^{T}\right]\right\},
$$

where $\mathbf{D}=\left[\left(\partial_{\mathbf{a}_{1}} / \partial_{f_{1}}\right),\left(\partial_{\mathbf{a}_{2}} / \partial_{f_{2}}\right) \cdots\left(\partial_{\mathbf{a}_{K}} / \partial_{f_{K}}\right),\left(\partial_{\mathbf{a}_{1}} / \partial_{\theta_{1}}\right)\right.$, $\left.\left(\partial_{\mathbf{a}_{2}} / \partial_{\theta_{2}}\right) \times \cdots\left(\partial_{\mathbf{a}_{K}} / \partial_{\theta_{K}}\right)\right]$, with $\mathbf{a}_{k}$ being the $k$ th column of A

$$
\begin{gathered}
\hat{\mathbf{P}}_{s}=\frac{1}{N} \sum_{t=1}^{N} \mathbf{s}_{t} \mathbf{s}_{t}^{H}, \\
\Pi_{\mathbf{A}}^{\perp}=\mathbf{I}_{P M}-\mathbf{A}\left(\mathbf{A}^{H} \mathbf{A}\right)^{-1} \mathbf{A}^{H} .
\end{gathered}
$$

3.4. Discussion. The performance of this proposed algorithm with nonuniform linear array will be discussed in this section. The distance between the $m$ th antenna and the left reference element is $d_{m}(m=0, \ldots, M-1)$, where $d_{0}=0$.

There is an array of $M$ sensors on which $K$ incident waves impinge $(M>K)$. The direction matrix $\mathbf{A}^{\prime}$ is

$$
\mathbf{A}^{\prime}=\left[\begin{array}{cccc}
1 & 1 & \cdots & 1 \\
e^{-j d_{1} \gamma_{1}} & e^{-j d_{1} \gamma_{2}} & \cdots & e^{-j d_{1} \gamma_{K}} \\
\vdots & \vdots & \ddots & \vdots \\
e^{-j d_{M-1} \gamma_{1}} & e^{-j d_{M-1} \gamma_{2}} & \cdots & e^{-j d_{M-1} \gamma_{K}}
\end{array}\right]
$$

where $\gamma_{k}=2 \pi f_{k} \sin \left(\theta_{k}\right) / c, k=1,2, \ldots, K$.

Replacing matrix A with $\mathbf{A}^{\prime}$ in (3)-(12), we can get the frequency estimation by (15).
For a nonuniform linear array, (20) and (21) can be rewritten as follows:

$$
\begin{gathered}
\mathbf{E}_{c 1}^{\prime}=\left[\begin{array}{c}
\mathbf{G} \\
\mathbf{G} \Phi_{1} \\
\vdots \\
\mathbf{G} \Phi_{M-2}
\end{array}\right] \boldsymbol{\Pi}=\left[\begin{array}{c}
\mathbf{E}_{c 11}^{\prime} \\
\mathbf{E}_{c 12}^{\prime} \\
\vdots \\
\mathbf{E}_{c 1(M-1)}^{\prime}
\end{array}\right], \\
\mathbf{E}_{c 2}^{\prime}=\left[\begin{array}{c}
\mathbf{G} \Phi_{1} \\
\mathbf{G} \Phi_{2} \\
\vdots \\
\mathbf{G} \Phi_{M-1}
\end{array}\right] \boldsymbol{\Pi}=\left[\begin{array}{c}
\mathbf{E}_{c 21}^{\prime} \\
\mathbf{E}_{c 22}^{\prime} \\
\vdots \\
\mathbf{E}_{c 2(M-1)}^{\prime}
\end{array}\right],
\end{gathered}
$$

where

$$
\begin{array}{r}
\boldsymbol{\Phi}_{j}=\operatorname{diag}\left\{e^{-j d_{j} \gamma_{1}}, e^{-j d_{j} \gamma_{2}}, \ldots, e^{-j d_{j} \gamma_{K}}\right\} \in \mathbf{C}^{K \times K}, \\
j=1,2, \ldots, M-1,
\end{array}
$$

$\mathbf{E}_{c 1 j}^{\prime}$ and $\mathbf{E}_{c 2 j}^{\prime}$ represent the $(j-1) P+1$ to $j P$ th rows of matrix $\mathbf{E}_{c 1}^{\prime}$ and $\mathbf{E}_{c 2}^{\prime}$, respectively.

We define

$$
\mathbf{C}_{j}=\mathbf{E}_{c 1 j}^{+} \mathbf{E}_{c 2 j}, \quad j=1,2, \ldots, M-1 .
$$

Then, the DOA estimation can be obtained via

$$
\begin{array}{r}
\hat{\theta}_{k}=\frac{1}{M-1} \sum_{j=1}^{M-1} \arcsin \left(\frac{c}{2 \pi f_{k}\left(d_{j}-d_{j-1}\right)} \operatorname{angle}\left(\xi_{k j}\right)\right), \\
k=1,2, \ldots, K,
\end{array}
$$

where $\xi_{k j}$ is the $k$ th diagonal element of matrix $\mathbf{C}_{j}$.

3.5. The Advantages of the Proposed Algorithm. The proposed algorithm has the following advantages.

(1) The proposed algorithm has a better angle estimation performance than Wang's ESPRIT algorithm.

(2) The proposed algorithm can obtain automatically paired parameter estimation, while Wang's ESPRIT algorithm requires additional pairing.

(3) The proposed algorithm has a comparative computational complexity in contrast to Wang's ESPRIT algorithm.

(4) This proposed algorithm also suit for nonuniform linear arrays.

\section{Simulation Results}

We present Monte Carlo simulations that are used to assess the angle and frequency estimation performance of this algorithm. The number of Monte Carlo trials is 1000. Note that $M$ is the number of antennas; $P$ is the number of the delays; $N$ is the number of snapshots; $K$ is the number of the sources. 


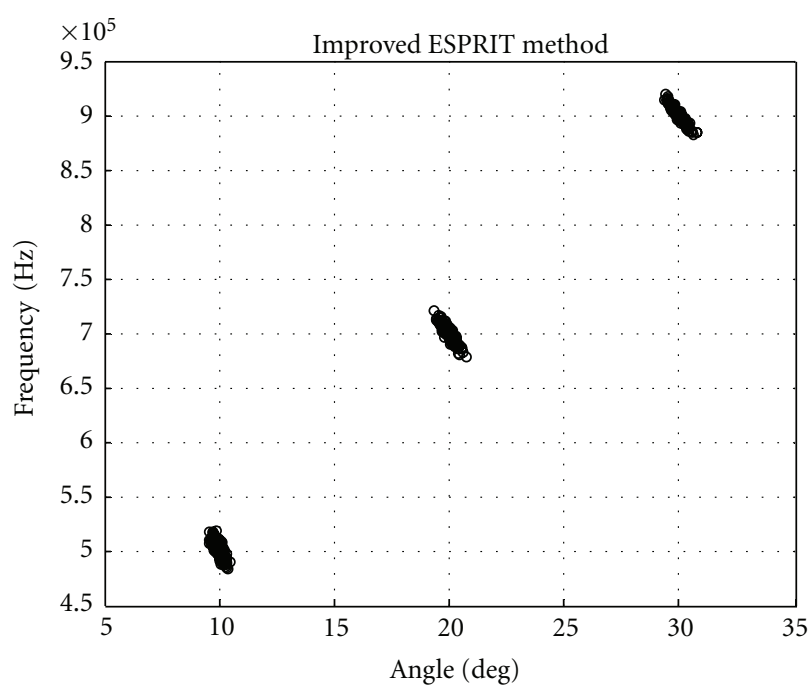

FIGURE 2: Angle-frequency scatter of proposed method, $\mathrm{SNR}=0 \mathrm{~dB}$.

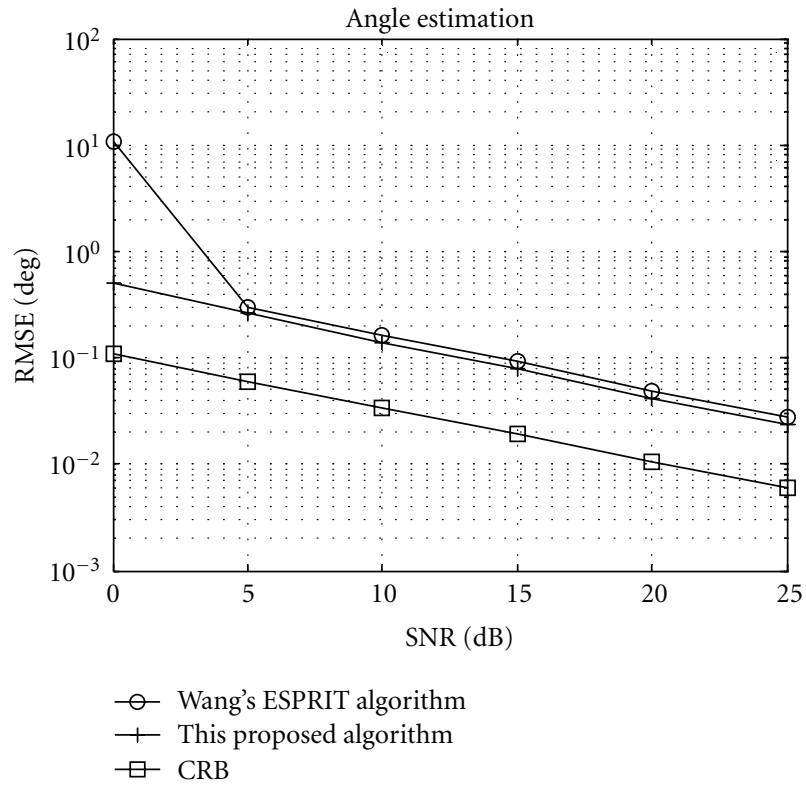

Figure 3: Angle estimation performance comparison at $M=12, K$ $=3, P=3$, and $N=100$.

\section{Define}

$$
\operatorname{RMSE}=\left(\frac{1}{K}\right) * \sum_{k=1}^{K} \sqrt{\left(\frac{1}{1000}\right) \sum_{m=1}^{1000}\left[a_{m k}-a_{0 k}\right]^{2}},
$$

where $a_{m k}$ is the $k$ th estimated angle/frequency, and $a_{0 k}$ is the $k$ th perfect angle/frequency.

Simulation 1. The performance of Wang's ESPRIT algorithm and this proposed algorithm is investigated. $M=12, K=3$, $P=3$, and $N=400$ in this simulation. Their DOAs are $10^{\circ}, 20^{\circ}$, and $30^{\circ}$, and their carrier frequencies are $500 \mathrm{kHz}$, $700 \mathrm{kHz}$, and $900 \mathrm{kHz}$. Figures 1 and 2 show the performance of Wang's ESPRIT algorithm and this proposed algorithm

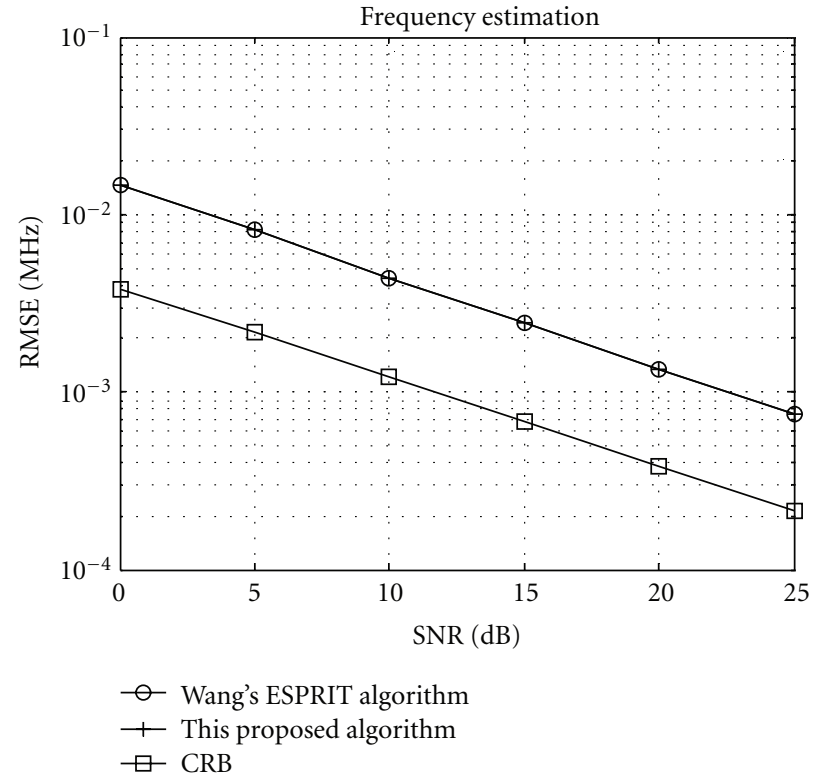

FIGURE 4: Frequency estimation performance comparison at $M=$ $12, K=3, P=3$, and $N=100$.

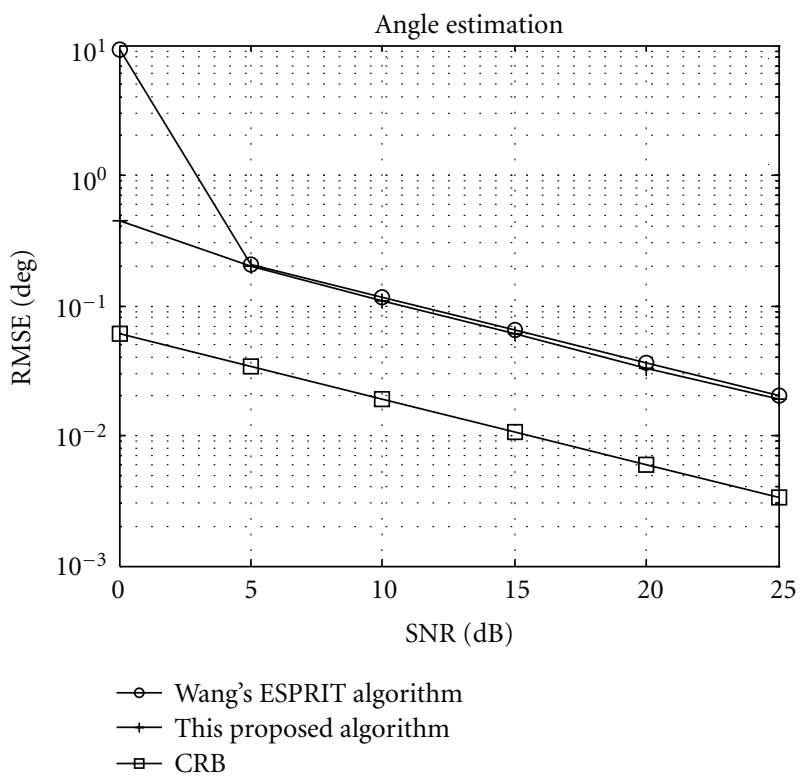

Figure 5: Angle estimation performance comparison at $M=12, K$ $=4, P=3$, and $N=400$.

with $\mathrm{SNR}=0 \mathrm{~dB}$. From Figures 1 and 2, we find the proposed algorithm can obtain automatically paired parameter estimation, while Wang's ESPRIT algorithm cannot, so it works well.

Simulation 2. We compare this proposed algorithm with Wang's ESPRIT algorithm and CRB. From Figures 3 and 4, we find that this proposed algorithm has better angle estimation performance than Wang's ESPRIT algorithm and has the same frequency estimation accuracy. Figure 5 to Figure 7 give more angle estimation performance comparison results 


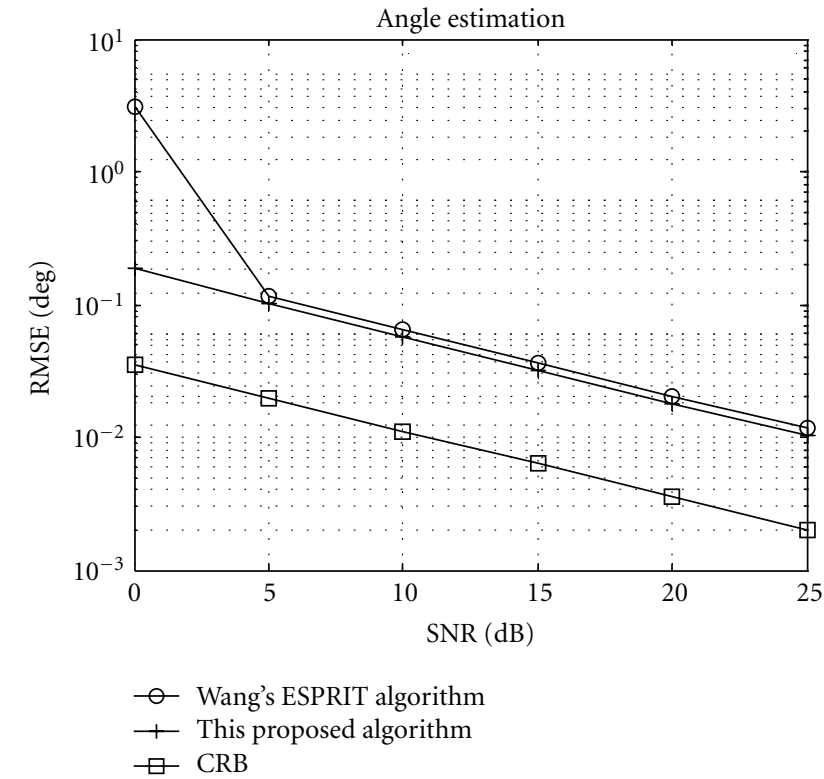

Figure 6: Angle estimation performance comparison at $M=16, K$ $=3, P=3$, and $N=400$.

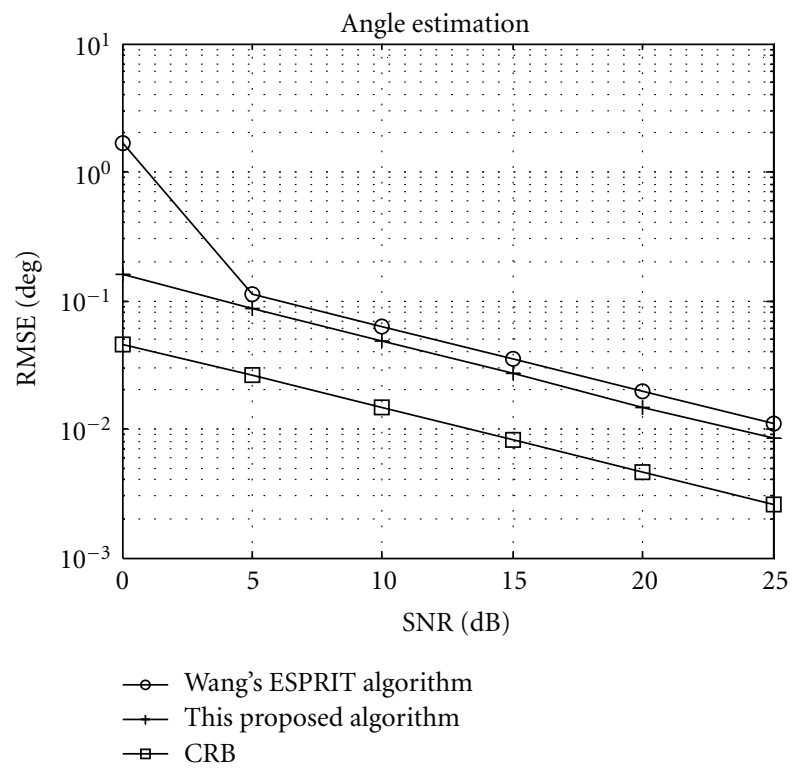

Figure 7: Angle estimation performance comparison at $M=12, K$ $=3, P=4$, and $N=400$.

between these two methods at different $M, K, P$, and $N$. Since the frequency estimation performance of the proposed is same with that of Wang's ESPRIT, it is not necessary to plot it from Figures 5, 6, and 7.

Simulation 3. This proposed algorithm performance under different snapshots $N$ is investigated in this simulation. $M=$ $12, K=3$, and $P=3$ are used in this simulation. Figure 8 shows the angle-frequency estimation performance under different $N$. We find that the angle-frequency estimation performance of this algorithm is improved with $N$ increasing.

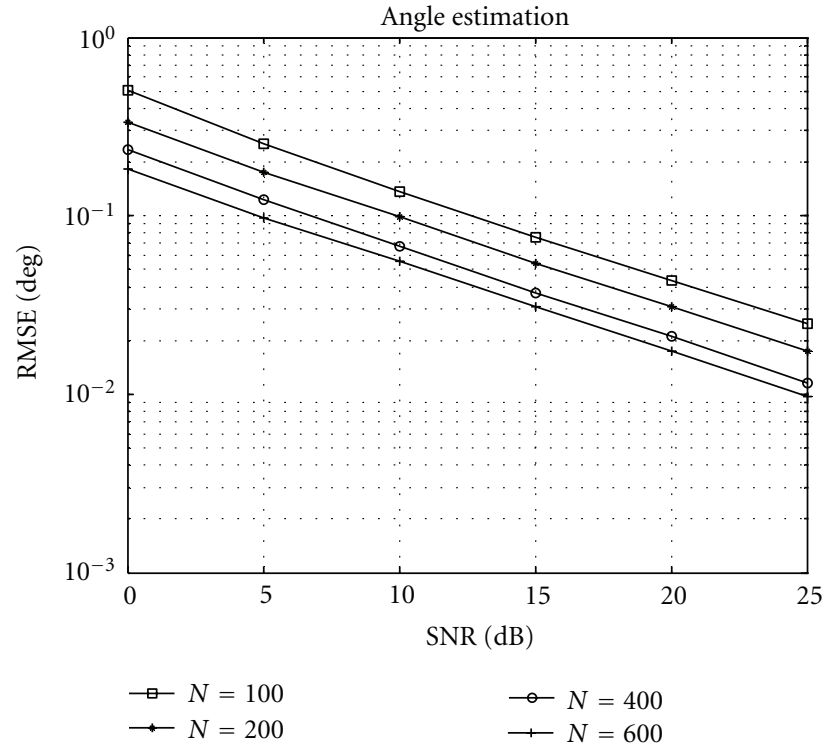

(a) Angle estimation

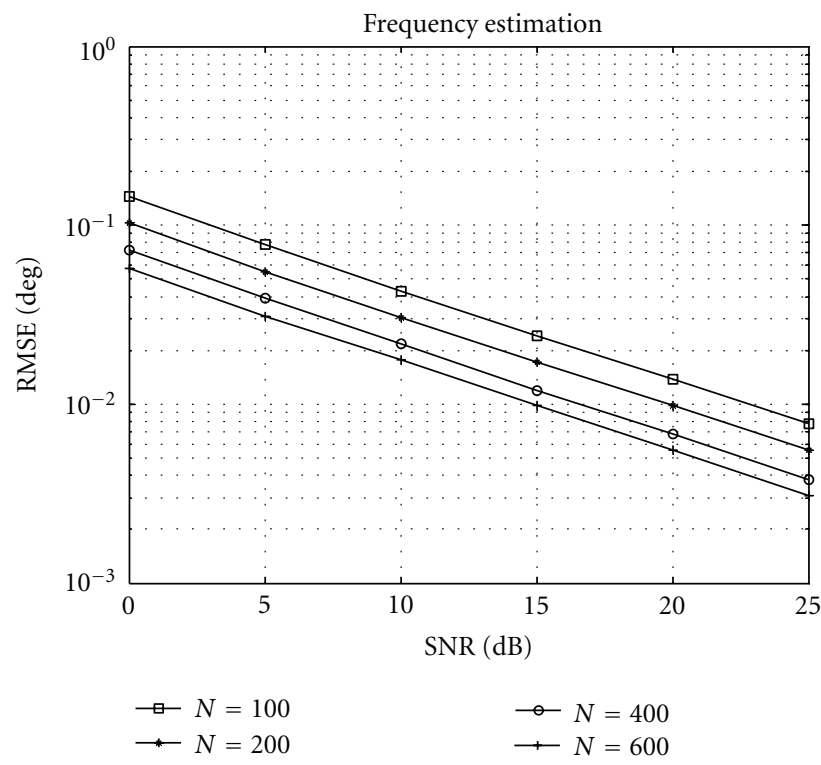

(b) Frequency estimation

FIGURE 8: Angle-frequency estimation with different snapshot $N$.

Simulation 4. The performance of this algorithm under different source number $K$ is investigated in the simulation. $M=12, P=3$, and $N=400$ are used in this simulation. The source number $K$ is set to 2,3 , and 4 . This proposed algorithm has different performance under different source numbers, as shown in Figure 9. From Figure 9, we find that angle and frequency estimation performance of this algorithm degrades with the increasing of the source number K.

Simulation 5. The performance of this algorithm under different antenna number $M$ is investigated in the simulation. 


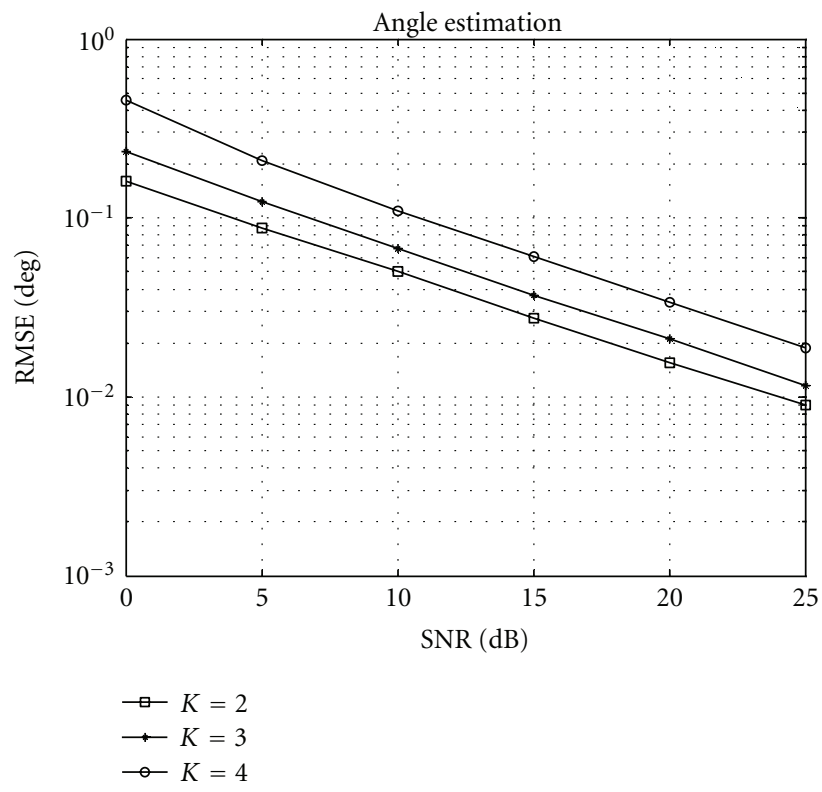

(a) Angle estimation

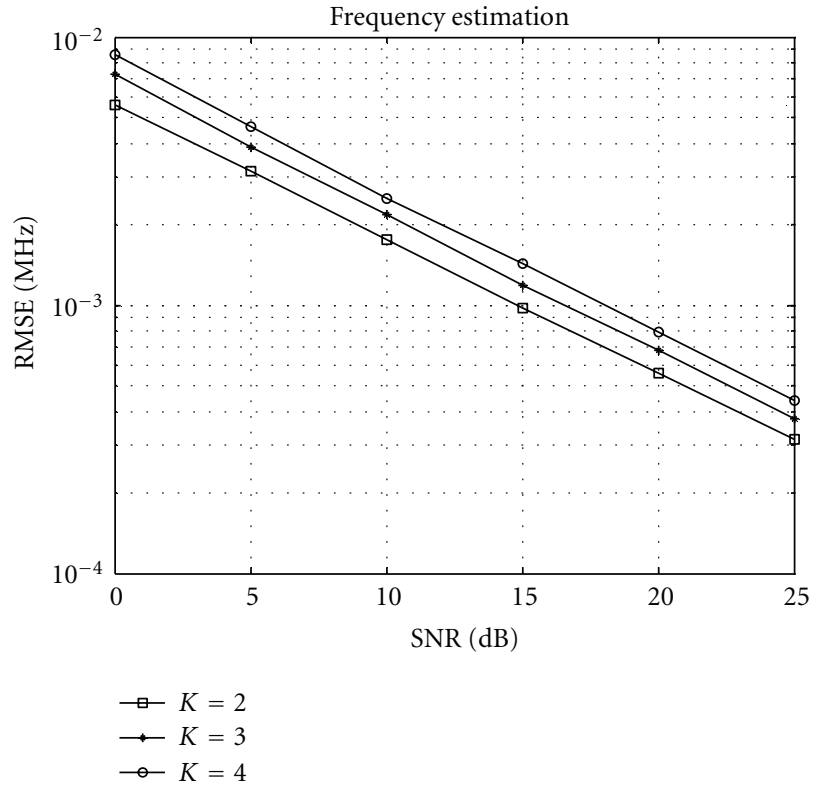

(b) Frequency estimation

Figure 9: Angle-frequency estimation with different sources.

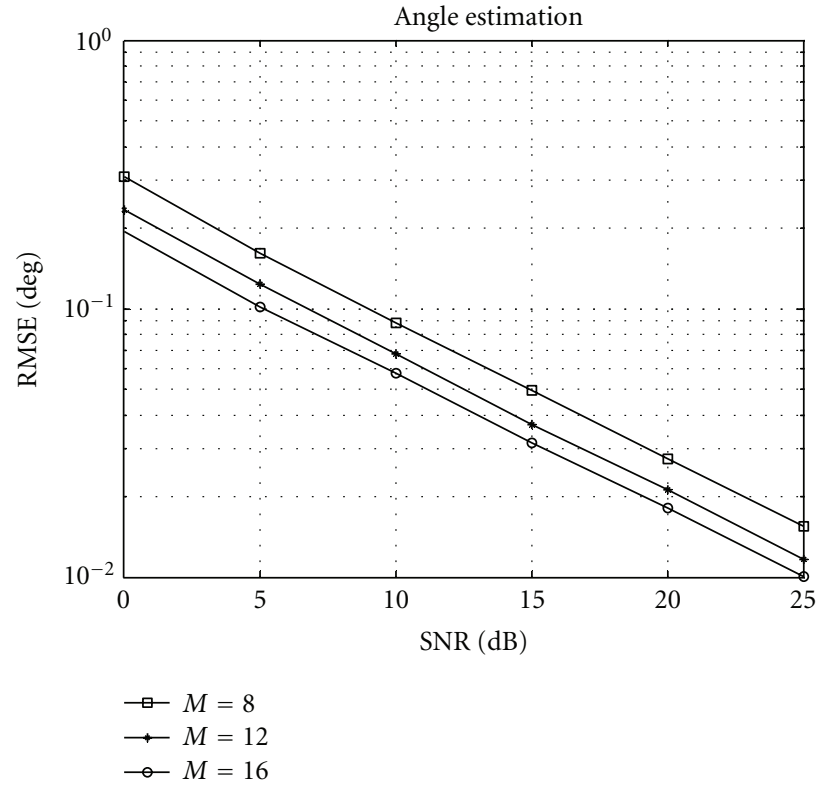

(a) Angle estimation

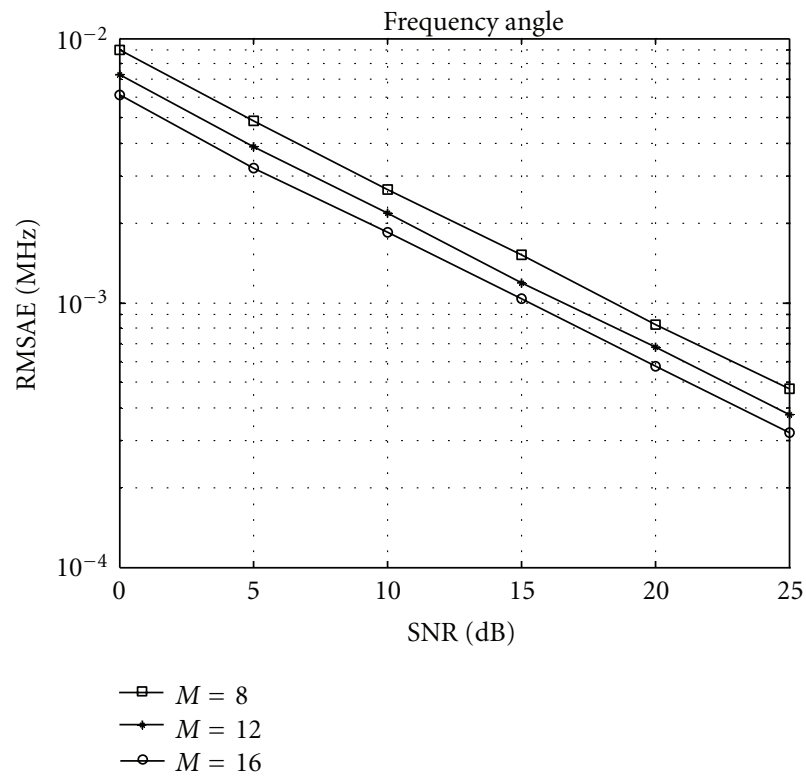

(b) Frequency estimation

Figure 10: Angle-frequency estimation with different antennas.

$K=3, P=3$, and $N=400$ are used in this simulation. The antenna number $M$ is set to 8,12 , and 16 . This proposed algorithm has different performance under different antenna numbers, as shown in Figure 10. From Figure 10, we find that angle and frequency estimation performance of this algorithm is improved with $M$ increasing.

Simulation 6. The performance of this algorithm under different delay number $P$ is investigated in the simulation.
$M=12, K=3$, and $N=400$ are used in this simulation. The delay number $P$ is set to 2, 3, 4, and 5. This proposed algorithm has different performance under different delay numbers, as shown in Figure 11. From Figure 11, we find that angle and frequency estimation performance of this algorithm is improved with $P$ increasing.

Simulation 7. The performance of this proposed algorithm with nonuniform linear arrays is investigated. $M=8, K=3$, 


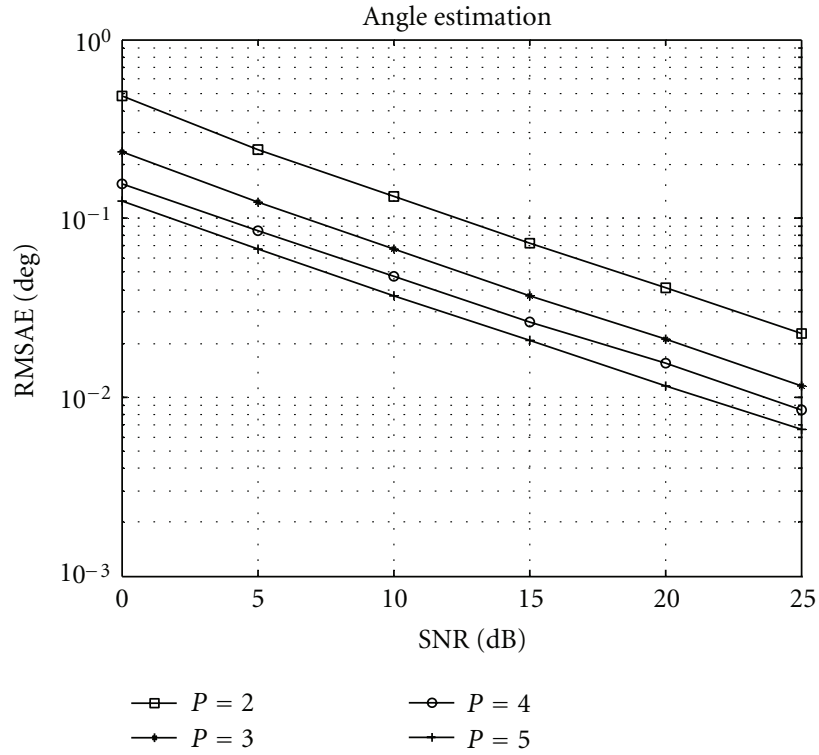

(a) Angle estimation

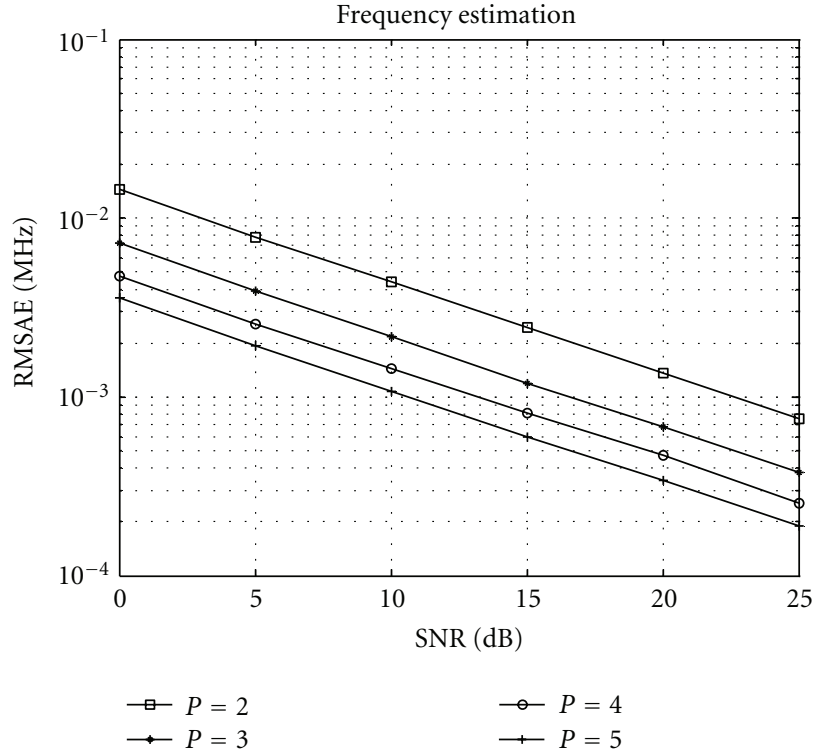

(b) Frequency estimation

FIGURE 11: Angle-frequency estimation with different delay number.

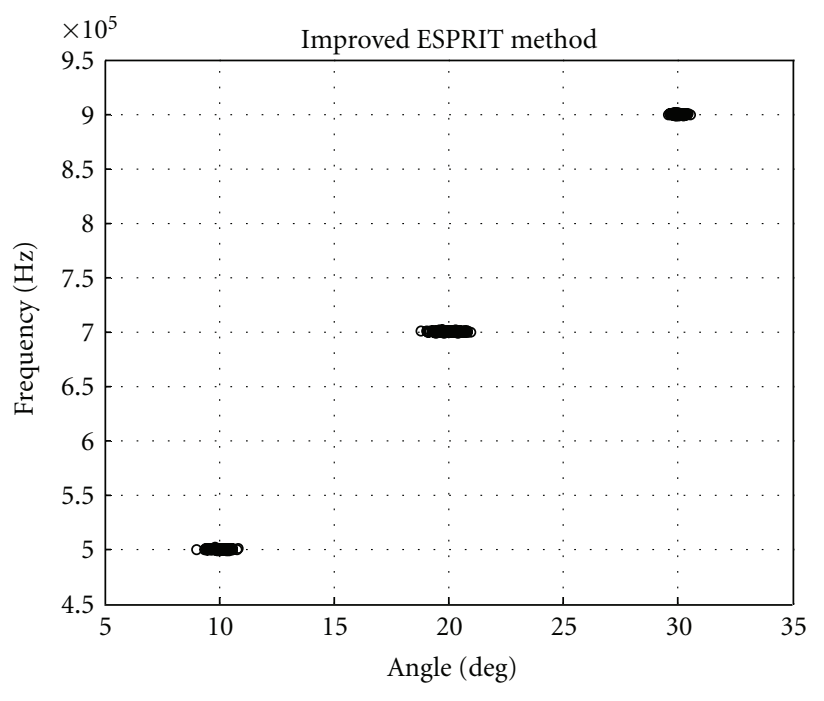

FIGURE 12: Angle-frequency scatter for nonuniform linear arrays, $\mathrm{SNR}=10 \mathrm{~dB}$.

$P=3$, and $N=400$ are used in this simulation. Their DOAs are $10^{\circ}, 20^{\circ}$, and $30^{\circ}$, and their carrier frequencies are $500 \mathrm{kHz}, 700 \mathrm{kHz}$, and $900 \mathrm{kHz}$. Figure 12, shows this proposed algorithm with nonuniform linear arrays with $\mathrm{SNR}=0 \mathrm{~dB}$. From Figure 12, we find the proposed algorithm can also work well in the case of nonuniform linear arrays.

\section{Conclusion}

This paper has presented an improved joint angle-frequency estimation method, which has better angle estimation performance than Wang's ESPRIT algorithm and has the same frequency estimation accuracy. The computational complexity of this proposed algorithm is comparative in contrast to Wang's ESPRIT algorithm. Since the DOA and frequency estimations suffer from the same permutation ambiguity, this novel method can obtain automatically paired DOA and frequency. This advantage is more obvious when the input SNR is below $0 \mathrm{~dB}$. Furthermore, the proposed algorithm can also work well in the case of nonuniform linear arrays.

\section{Acknowledgments}

This paper is supported by China NSF Grant (61201208), Aeronautical Science Foundation of China (2009ZC52036), Nanjing University of Aeronautics and Astronautics Research Funding (NN2012068), and the Fundamental Research Funds for the Central Universities (NZ2012010, kfjj120115, kfjj20110215).

\section{References}

[1] X. Liu and G. Liao, "Direction finding and mutual coupling estimation for bistatic MIMO radar," Signal Processing, vol. 92, no. 2, pp. 517-522, 2012.

[2] J. Zhang, H. Wang, and X. Zhu, "Adaptive waveform design for separated transmit/receive ULA-MIMO radar," IEEE Transactions on Signal Processing, vol. 58, no. 9, pp. 4936-4942, 2010.

[3] V. Varadarajan and J. Krolik, "Array shape estimation and tracking using active sonar reverberation," IEEE Transactions on Aerospace and Electronic Systems, vol. 40, no. 3, pp. 10731086, 2004.

[4] X. Zhang, X. Gao, and D. Xu, "Multi-invariance ESPRIT-based blind DOA estimation for MC-CDMA with an antenna array," IEEE Transactions on Vehicular Technology, vol. 58, no. 8, pp. 4686-4690, 2009. 
[5] J. X. Wu, T. Wang, Z. Y. Suo, and Z. Bao, "DOA estimation for ULA by spectral Capon rooting method," Electronics Letters, vol. 45, no. 1, pp. 84-85, 2009.

[6] B. H. Wang, Y. L. Wang, H. Chen, and X. Chen, "Robust DOA estimation and array calibration in the presence of mutual coupling for uniform linear array," Science in China, Series F, vol. 47, no. 3, pp. 348-361, 2004.

[7] Y. Zhang and Z. Ye, "Efficient method of DOA estimation for uncorrected and coherent signals," IEEE Antennas and Wireless Propagation Letters, vol. 7, pp. 799-802, 2008.

[8] X. Zhang and D. Xu, "Improved coherent DOA estimation algorithm for uniform linear arrays," International Journal of Electronics, vol. 96, no. 2, pp. 213-222, 2009.

[9] X. Zhang, J. Yu, G. Feng, and D. Xu, "Blind direction of arrival estimation of coherent sources using multi-invariance property," PIER: Progress in Electromagnetics Research, vol. 88, pp. 181-195, 2008.

[10] E. Rosnes and A. Vahlin, "Frequency estimation of a single complex sinusoid using a generalized Kay estimator," IEEE Transactions on Communications, vol. 54, no. 3, pp. 407-415, 2006.

[11] J. Tsui, Digital Techniques for Wideband Receivers, chapter 12, Artech House, Norwood, Mass, USA, 2nd edition, 2001.

[12] M. Djeddou, A. Belouchrani, and S. Aouada, "Maximum likelihood angle-frequency estimation in partially known correlated noise for low-elevation targets," IEEE Transactions on Signal Processing, vol. 53, no. 8, pp. 3057-3064, 2005.

[13] M. D. Zoltowski and C. P. Mathews, "Real-time frequency and 2-D angle estimation with sub-Nyquistspatio-temporal sampling," IEEE Transactions on Signal Processing, vol. 42, no. 10, pp. 2781-2794, 1994.

[14] M. Haardt and J. A. Nossek, "3-D unitary ESPRIT for joint 2-D angle and carrier estimation," in Proceedings of the IEEE International Conference on Acoustics, Speech, and Signal Processing (ICASSP '97), pp. 255-258, Munich, Germany, April 1997.

[15] A. N. Lemma, A. J. van der Veen, and E. F. Deprettere, "Joint angle-frequency estimation using multi-resolution ESPRIT," in Proceedings of the IEEE International Conference on Acoustics, Speech and Signal Processing (ICASSP '98), pp. 1957-1960, Seattle, Wash, USA, May 1998.

[16] A. N. Lemma, A. J. Van Der Veen, and E. F. Deprettere, "Analysis of joint angle-frequency estimation using ESPRIT," IEEE Transactions on Signal Processing, vol. 51, no. 5, pp. 1264$1283,2003$.

[17] S. Wang and X. Zhou, "Direction-of-arrival and frequency estimation in array signal processing," Journal of Shanghai Jiaotong University, vol. 33, no. 1, pp. 40-42, 1999.

[18] W. Xudong, "Joint angle and frequency estimation using multiple-delay output based on ESPRIT," EURASIP Journal on Advances in Signal Processing, vol. 2010, Article ID 358659, 6 pages, 2010.

[19] P. Stoica and A. Nehorai, "Performance study of conditional and unconditional direction-of-arrival estimation," IEEE Transactions on Acoustics, Speech, and Signal Processing, vol. 38, no. 10, pp. 1783-1795, 1990. 

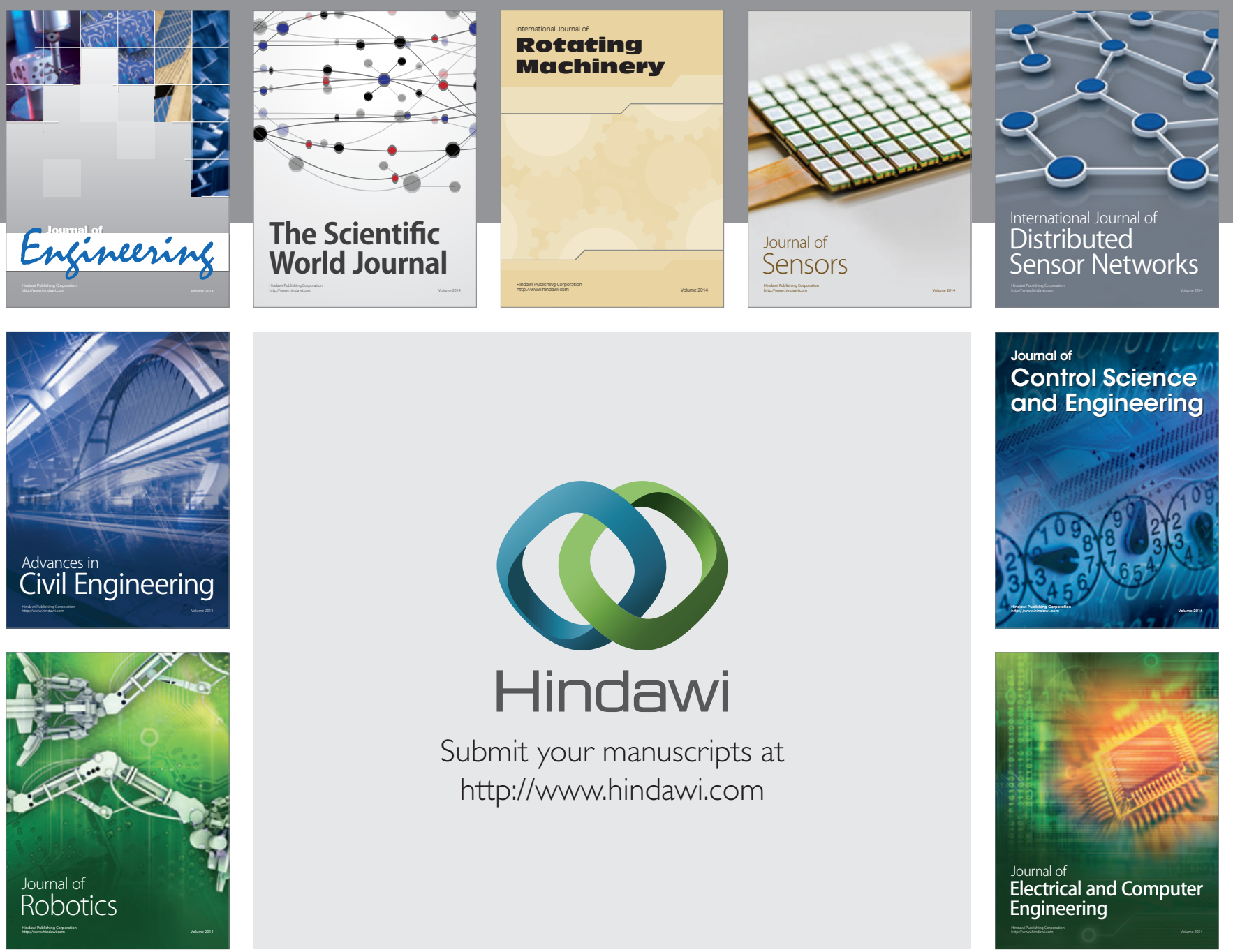

Submit your manuscripts at

http://www.hindawi.com
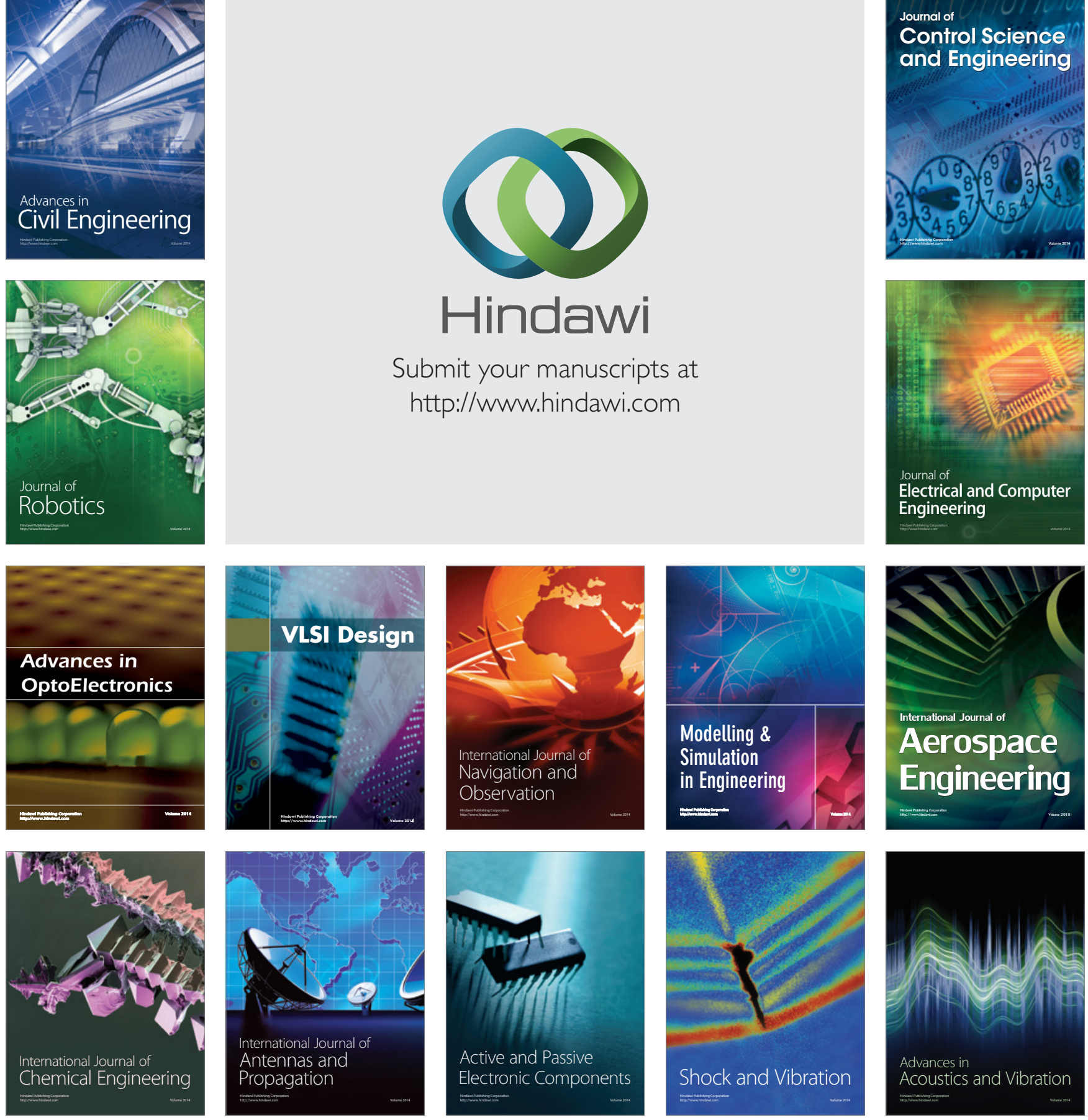